\title{
Significance of PSA Screening in Niigata, Japan: Survey of Actual Status of New Cases of Prostate Cancer
}

\author{
Toshihiro Saito,' \\ Shuichi Komatsubara, ${ }^{2}$ \\ Noboru Hara, ${ }^{3}$ Tsutomu Nishiyama, ${ }^{3}$ \\ Akiyoshi Katagiri, ${ }^{4}$ Fumio Ishizaki, ${ }^{5}$ \\ Akihiko Hatano, ${ }^{6}$ \\ Takeshi Komeyama, ${ }^{7}$ Shugo Hanyu, ${ }^{8}$ \\ Masahiro Terunuma, \\ Tomoyuki Imai, ${ }^{10}$ Hideto Go, ${ }^{11}$ \\ Hiroaki lida, ${ }^{12}$ Shigenori Kurumada, ${ }^{13}$ \\ Shunsuke Yamaguchi, ${ }^{4,14}$ \\ Keisuke Takeda, ${ }^{5,15}$ Takashi Ando, ${ }^{14,15}$ \\ Tatsuhiko Hoshii, ${ }^{5}$ Takashi Kasahara, ${ }^{5}$ \\ Yoshihiko Tomita $^{5}$ \\ 'Department of Urology, Niigata Cancer \\ Center Hospital, Niigata, Japan; \\ ${ }^{2}$ Department of Urology, Niigata Minami \\ Hospital, Niigata, Japan; ${ }^{3}$ Department of \\ Urology, Uonuma Institute of Community \\ Medicine, Niigata University Medical and \\ Dental Hospital, Minamiuonuma, Japan; \\ ${ }^{4}$ Department of Urology, Niigata Prefectural \\ Central Hospital, Joetsu, Japan; \\ ${ }^{5}$ Department of Renal and Urinary \\ Pathology, Graduate School of Medical and \\ Dental Sciences, Niigata University, Niigata, \\ Japan; ${ }^{6}$ Department of Urology, Niigata \\ Prefectural Shibata Hospital, Shibata, Japan; \\ ${ }^{7}$ Department of Urology, Nagaoka Red \\ Cross Hospital, Nagaoka, Japan; \\ ${ }^{8}$ Department of Urology, Kashiwazaki \\ General Hospital and Medical Center, \\ Kashiwazaki, Japan; 9 Department of \\ Urology, Nagaoka Chuo General Hospital, \\ Nagaoka, Japan; ${ }^{10}$ Department of Urology, \\ Niigata City General Hospital, Niigata, \\ Japan; "Department of Urology, Niigata \\ Saiseikai Sanjo Hospital, Sanjo, Japan; \\ ${ }^{12}$ Department of Urology, Joetsu General \\ Hospital, Joetsu, Japan; ${ }^{13}$ Department of \\ Urology, Saiseikai Niigata Hospital, Niigata, \\ Japan; ${ }^{14}$ Department of Urology, Tachikawa \\ General Hospital, Nagaoka, Japan; \\ ${ }^{15}$ Department of Urology, Murakami \\ General Hospital, Murakami, Japan
}

Correspondence: Toshihiro Saito

Department of Urology, Niigata Cancer

Center Hospital, 2-15-3 Kawagishi-cho,

Niigata, 95I-8566, Japan

Tel +8I-25-266-5 III

Fax +8I-25-233-3849

Email urotoshi@niigata-cc.jp
Purpose: This study aims to investigate the utility of prostate-specific antigen (PSA) screening by conducting an all-case survey of newly diagnosed prostate cancer patients at Niigata Prefecture, Japan.

Patients and Methods: Depending on whether patients were subjected to screening, information was prospectively collected on all prostate cancer patients newly diagnosed between October 1, 2019, and September 30, 2020, at all institutions in Niigata Prefecture where urologists performing prostate biopsy routinely work and differences in clinical parameters were investigated.

Results: PSA was measured in 478 out of 1332 patients $(35.8 \%)$ as part of a community health screening. The rate of metastatic carcinoma (M1) in all patients was $14.9 \%$. When patients were divided into three categories of population-based screening (community health screening and workplace health screening), opportunistic screening (PSA measurements at complete medical check-ups or on patient request), and testing triggered by clinical symptoms or findings, the proportion of metastatic cancer was $4.5 \%, 3.7 \%$, and $30.6 \%$, respectively, demonstrating that the number of distant metastases was significantly lesser in all patients who underwent screening.

Conclusion: The one-year all-case survey of newly diagnosed prostate cancer patients demonstrated that PSA screening significantly contributed to the early diagnosis of current prostate cancer in Japan.

Keywords: prostate cancer, PSA screening, population-based screening, opportunistic screening, one-year all-case survey

\section{Introduction}

More than 10 years have passed since the "Japanese Guideline for Prostate Cancer Screening" from The Research Group for Cancer Screening Guidelines supported by a 2007 Grand-in-Aid for Cancer Research from the Japanese Ministry of Health, Labour and Welfare was published in 2008. ${ }^{1}$ This guideline states that "Prostate cancer screening using either DRE or PSA is not recommended for population-based screening due to insufficient evidence (Recommendation grade I)".

Recently, on the front page of the morning edition of Nihon Keizai Shimbun (Japanese newspaper) on November 23, 2019, an article criticizing the out-ofguideline examinations of local governments was published under the headline " $90 \%$ of municipalities conduct cancer screenings with poor evidence". With the description of PSA screening for prostate cancer is a large part of this article, this demonstrates that the influence of the guideline is still strong even today. 
However, the effect of PSA screening in reducing mortality has already been demonstrated in randomized controlled studies (RCTs) and practical prospective observational studies in Europe, ${ }^{2-5}$ and the 2018 Screening Guideline for Prostate Cancer from the Japanese Urological Association states that PSA testing-based prostate cancer screening has been demonstrated to reduce prostate cancer mortality. ${ }^{6}$

One study, the PLCO study in the United States, failed to demonstrate a reduction in mortality with PSA screening. ${ }^{7,8}$ However, this study had a highly contaminated control group (even in the control group, most of the patients actually underwent PSA testing), which resulted in the mortality not being reported to decrease in the screening group. ${ }^{9}$ At that time in the United States, there was a high interest in the early detection of prostate cancer and there were sufficient opportunities for PSA to be measured without having to implement mass screening; thus, the significance of mass screening could not be demonstrated. At the very least, this is not the rationale for ruling out prostate screening in Japan.

When considering the significance of PSA screening in Japan, the ideal method to demonstrate the effect of reducing the mortality rate would be using RCTs. However, it would be ethically unacceptable to conduct RCTs under the current situation where screening is already widespread. Thus, to investigate how much PSA screening contributes to the medical practice and treatment of prostate cancer in Japan, an all-case survey was conducted in newly discovered prostate cancer patients in Niigata Prefecture for one year, and the actual status was confirmed.

\section{Patients and Methods}

\section{Study Population and Patient Selection}

This study was carried out following the ethical standards of the Declaration of Helsinki. The ethics review boards of the Niigata Cancer Center Hospital (approval number: 2019-2) and all participating hospitals approved the protocol. Informed consent was obtained in the form of opt-out on the bulletin boards and websites.

We included all prostate cancer patients newly diagnosed between October 1, 2019, and September 30, 2020, at all institutions in Niigata Prefecture where urologists performing routine prostate biopsies work. Inclusion criteria were 1) newly histologically proven prostate cancer and 2) the prostate cancer patients who started treatment based on a clinical diagnosis without biopsy. Exclusion criteria were insufficient baseline information including the serum PSA value or the history of initial PSA measurement. Information was prospectively collected to clarify whether these patients underwent screening.

\section{Triggers for PSA Measurement}

Attention was focused on whether or not the initial PSA measurement in each patient was triggered by screening examinations and patients were purposely asked which of the following six categories they met: "Community health screening (regardless of the timing of screening or the presence or absence of symptoms)," "Workplace health screening (regardless of the timing of screening or the presence or absence of symptoms)," "Complete medical check-up (regardless of the timing of the examination and the presence or absence of symptoms)," "Requested PSA measurement without clinical findings or symptoms (other than health screening)," "PSA measurement in the asymptomatic patient based on some findings such as urinary occult blood or imaging," or "PSA measurement in the patient with symptoms such as voiding symptoms, gross hematuria, or symptoms of metastatic lesions." The results were tabulated together with clinical parameters such as PSA, stage, and Gleason score.

\section{Statistical Analyses}

Statistical analyses were performed using Chi-squared tests and $p<0.05$ was considered significant. All of the statistical analyses were carried out using the Statview 5.0 software program (Abacus Concepts, Berkley, CA USA).

\section{Results}

\section{Patient Characteristics}

There were 1336 patients newly diagnosed with prostate cancer during the one-year survey period. Among these, two patients were incidentally detected during radical cystectomy for bladder cancer, and PSA measurement was not performed before surgery. Moreover, in two patients, the history of PSA measurement was unclear. Excluding these 4 patients, 1332 patients were included in the present analysis. The patient demographics are shown in Table 1. Magnetic resonance imaging (MRI) was performed for staging in 1110 patients $(83.3 \%)$, computed tomography (CT) in 1268 (95.2\%) and bone scintigraphy in $1148(86.2 \%)$. 
Table I Patient Characteristics $(n=1332)$

\begin{tabular}{|l|l|l|}
\hline $\begin{array}{l}\text { Median Age, Years (IQR) } \\
\text { Median PSA, ng/mL (IQR) }\end{array}$ & 73 & $(69-79)$ \\
Tumor Stage (no. of cases) & 11.73 & $(7.29-25.06)$ \\
TlabN0M0 & 30 & \\
TIcN0M0 & 225 & $(2.3 \%)$ \\
T2N0M0 & 643 & $(16.9 \%)$ \\
T3N0M0 & 146 & $(48.3 \%)$ \\
T4NOM0 & 9 & $(11.0 \%)$ \\
TanyNIM0 & 46 & $(0.7 \%)$ \\
TanyNanyMI & 199 & $(3.5 \%)$ \\
Unknown & 34 & $(14.9 \%)$ \\
Gleason score (no. of cases) & & $(2.6 \%)$ \\
56 & 180 & \\
7 & 510 & $(13.5 \%)$ \\
$\leq 8$ & 549 & $(38.3 \%)$ \\
Unknown & 8 & $(41.2 \%)$ \\
No biopsy & 85 & $(6.6 \%)$ \\
\hline
\end{tabular}

Abbreviation: IQR, interquartile range.

\section{Triggers for PSA Measurement}

Figure 1 shows the triggers for PSA measurement. PSA measurement was performed in $478(35.8 \%)$ of the 1332 patients after community health screening. There were 34 in whom it was not feasible to distinguish between community health screening and workplace health screening. The results demonstrated that there were more patients who underwent testing because of community health screening than those tested because of symptoms.

\section{Metastatic Cancer Rate}

The rate of metastatic carcinoma (M1) in all patients was $14.9 \%$. Comparisons were made between three categories: population-based screening (community health screening and workplace health screening), opportunistic screening
(PSA measurements at complete medical health check-ups or at the patient's request), and testing triggered by symptoms or findings (Figure 2). Distant metastases were present in $30.6 \%$ of the patients who underwent testing triggered based on symptoms or results; however, this percentage was significantly lower in all patients who underwent screening ( $5 \%$ or less).

\section{PSA Values}

Comparisons of PSA values were performed in the three categories (Figure 3). PSA was $<20 \mathrm{ng} / \mathrm{mL}$ in $>85 \%$ of the patients who underwent population-based screening or opportunistic screening.

\section{Gleason Score}

Gleason scores were compared (Figure 4). In patients who underwent population-based screening or opportunistic screening, there were a significantly high number of patients with a Gleason score of 7 or less.

\section{Discussion}

Niigata Prefecture has a population of 2.2 million and has a land area approximately 5.74-fold larger than Tokyo, which is ranked fifth among the 47 prefectures in Japan. In the late 1880 s, the population of the prefecture was the largest in Japan for a certain time. However, after reaching a peak of 2.49 million in 1997, the number has been decreasing.

Prostate cancer screening in Niigata Prefecture was implemented in 2000. In 2002, a Prostate Cancer Screening Subcommittee was organized within the Cancer Management Committee of the Niigata Medical Association and the implementation of screening was

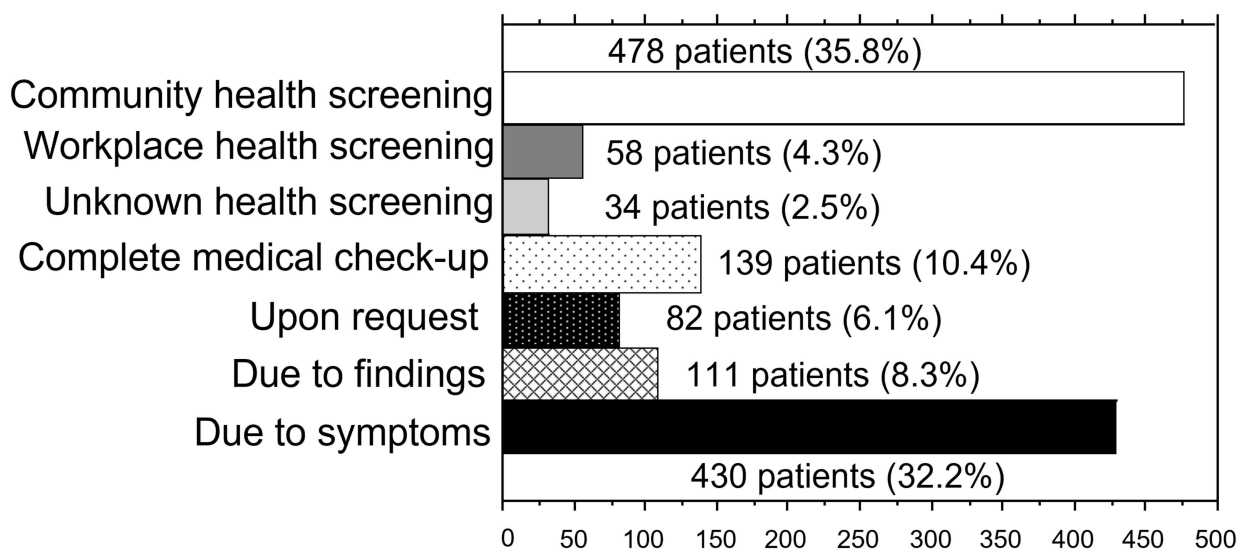

Figure I Triggers for measuring PSA $(n=\mid 332)$. 


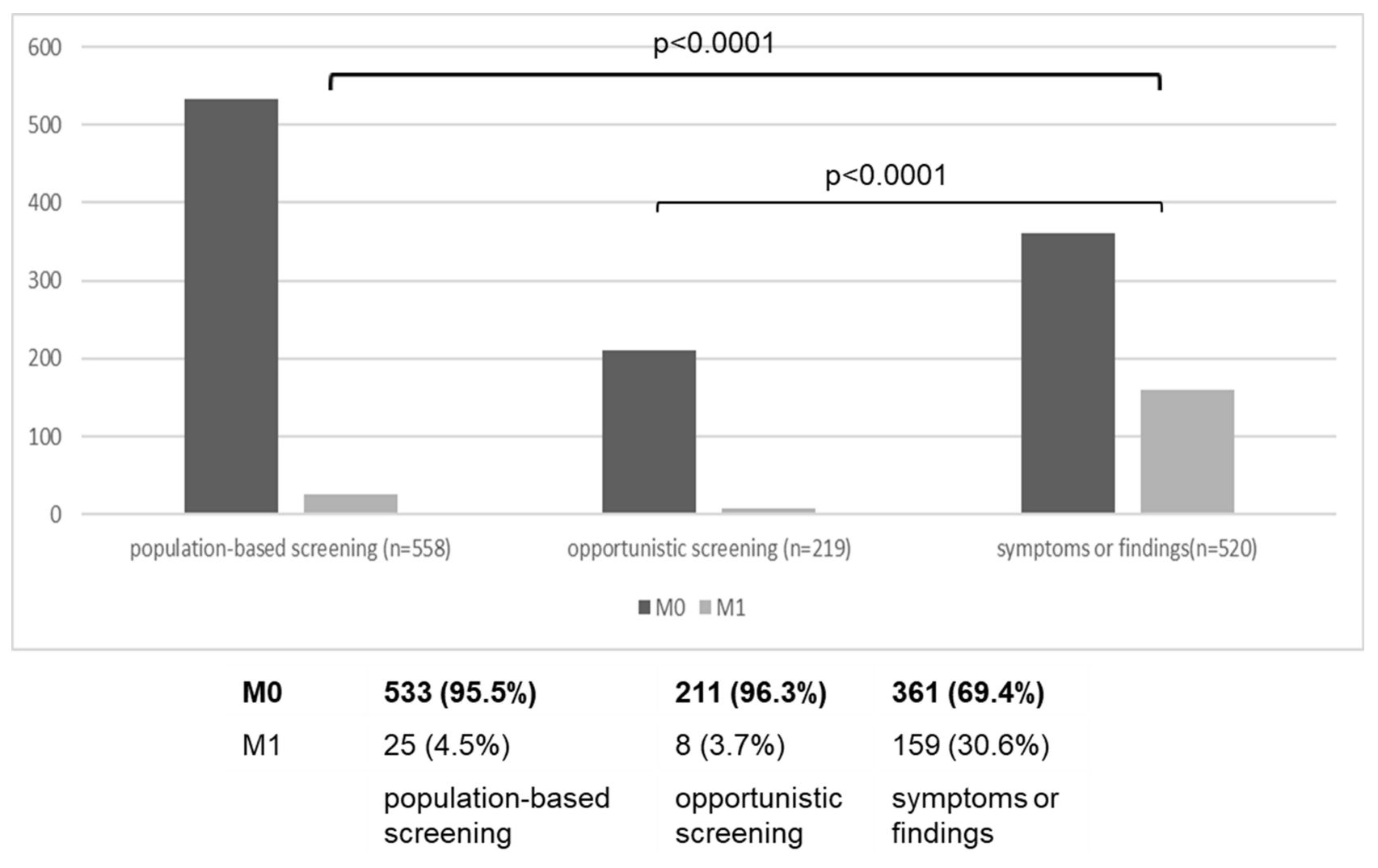

Figure 2 Metastatic cancer rate, excluding 35 patients with unknown staging $(n=1297)$.

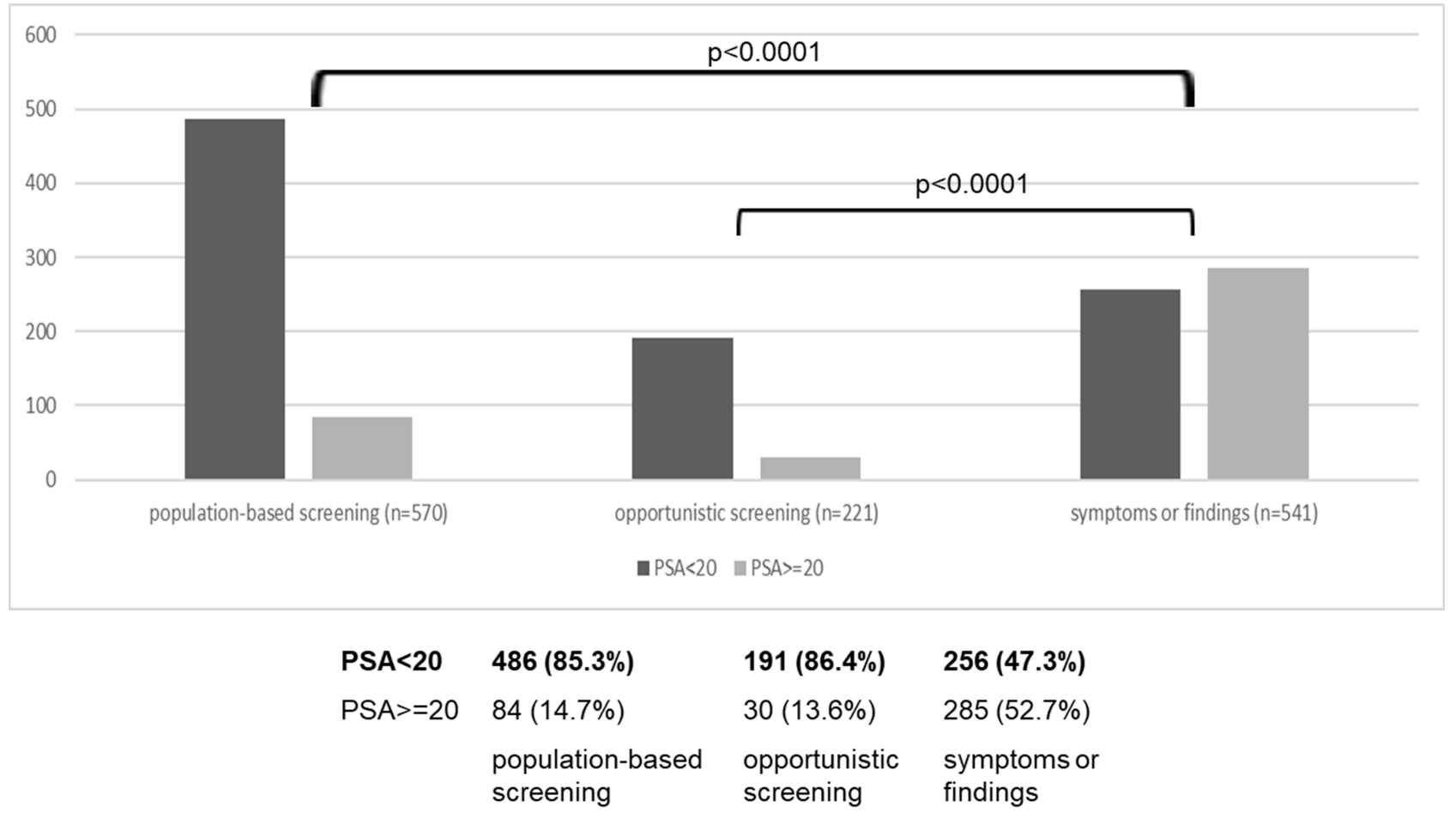

Figure 3 PSA values $(n=1332)$ 


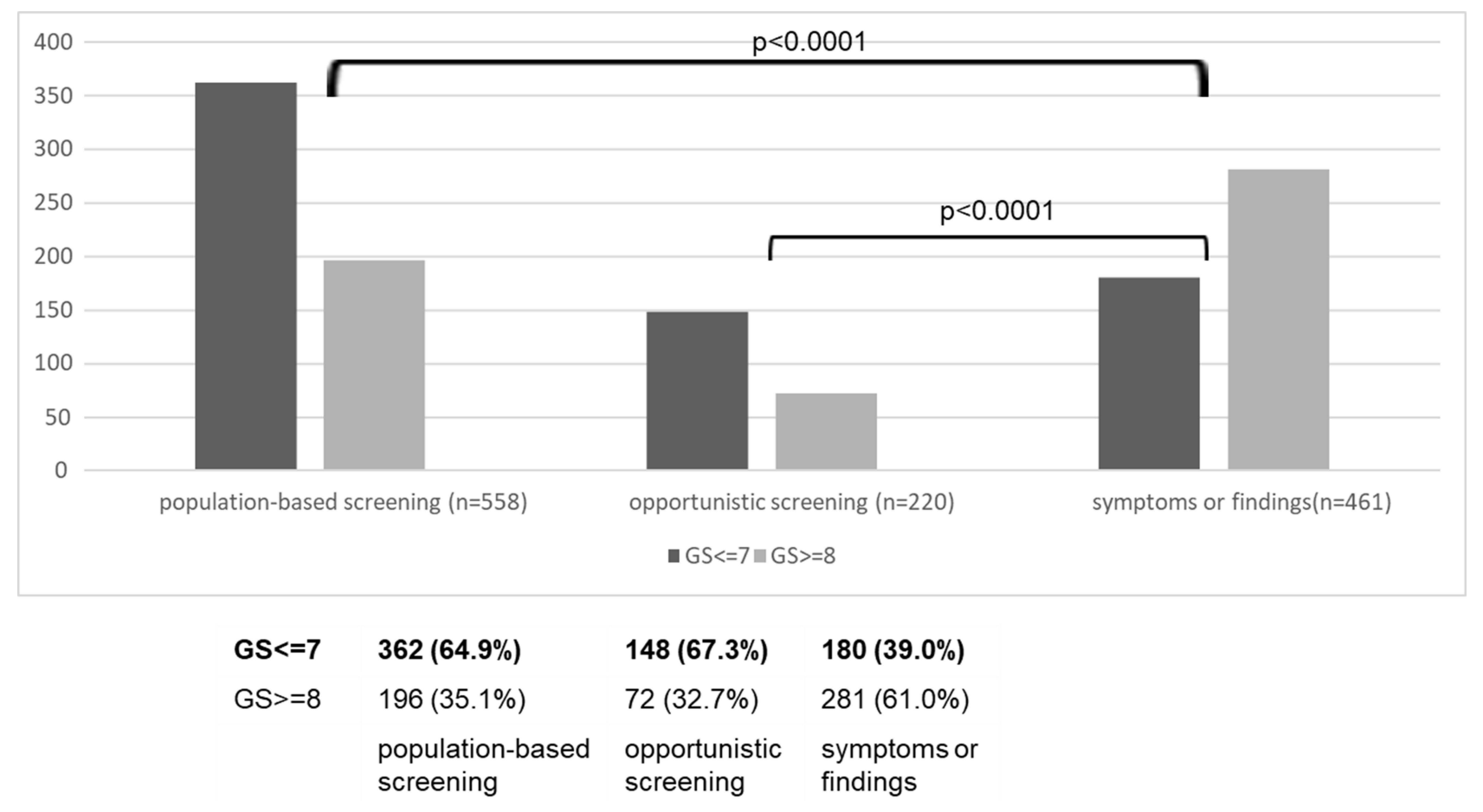

Figure 4 Gleason score, excluding 93 patients with unknown Gleason score $(n=1239)$.

started as a health project at the municipality level. In 2004, prostate cancer screening guidelines were published by the Prefectural Welfare and Health Department, the Prefectural Medical Association, and the Prefectural Association for Prevention of Adult Diseases as "References," and the Prostate Cancer Review Committee of the Prefectural Medical Association was established as a way to expand the aforementioned subcommittee, with prostate cancer screening conducted under standardized guidelines in the prefecture. ${ }^{10}$

PSA screening is implemented as a part of the Community Health Basic Screening System, with the target population being those 50 years or older. The cutoff values for the primary screening are stratified by age group: $3.0 \mathrm{ng} /$ $\mathrm{mL}$ for $50-64$ years, $3.5 \mathrm{ng} / \mathrm{mL}$ for $65-69$ years, $4.0 \mathrm{ng} / \mathrm{mL}$ for $\geq 70$ years, and $7.0 \mathrm{ng} / \mathrm{mL}$ for $\geq 80$ years.

According to statistics in Niigata Prefecture in 2019, 28 out of 30 municipalities participated, with the screening rate at $10.1 \%$ and a cancer detection rate of 403.5 (per 100,000). ${ }^{11}$

There is a standardized format for prostate cancer screening in Niigata Prefecture and most of the urologists in Niigata Prefecture were trained at the Urology Department of Niigata University, which provides an environment for highly effective all-case surveys from the perspective of clinical practice.
Although this survey aimed to show the effectiveness of PSA screening, it was unexpectedly difficult to distinguish between "Screened" and "Non-screened" in newly diagnosed prostate cancer patients. This is because screening does not always necessarily lead to a definitive diagnosis including prostate biopsy.

High PSA detected during a screening examination may be followed up without a biopsy for a certain time period. For example, in case of the patient in the gray zone PSA $(4-10 \mathrm{ng} / \mathrm{mL})$, the diagnosis is often made during an examination several years after the initial screening. In these instances, it is difficult to determine if the initial PSA test triggered the definitive diagnosis of cancer, and a retrospective review makes it difficult to determine whether such patients have been "Screened" or "Non-screened." If early cancer is detected after being followed for several years because of a prior screening, this should still be considered screening-detected prostate cancer and patients should be categorized as "Screened patients" even if the patient did not undergo the screening recently.

From the above point of view, an attempt was made to conduct a prospective survey of newly diagnosed patients in one year by purposely recording the information by interview and paying attention to whether the initial PSA measurement was triggered from screening or not. 
In this survey, triggers for PSA measurements were categorized into the following six categories: "Community health screening," "Workplace health screening," "Complete medical check-up," "Requested PSA measurement without clinical findings or symptoms (other than health screening)", "PSA measurement in the asymptomatic patient based on clinical findings", or "PSA measurement in the patient with symptoms".

The "population-based screening" mentioned in the guidelines from the Ministry of Health, Labour and Welfare are clearly defined in the guidelines as referring to activities conducted by organizations in charge of cancer management such as municipal governments, workplace areas, and health insurance societies. Thus, if these guidelines are actively considered, the effectiveness of "community health screening" plus "workplace health screening" should be discussed.

Because "complete medical check-ups and comprehensive health check-ups conducted at specialist screening institutions and medical institutions" and "screening tests for cancer recommended by family doctors for outpatients with chronic diseases" are listed as specific examples of opportunistic screening, "Complete medical check-ups" and "Requested PSA measurement without clinical findings or symptoms" in this survey were included in the category of "Opportunistic screening."

"Population-based screening" and "Opportunistic screening" are collectively referred to herein as "Widespread PSA screening,". The effectiveness of this "Widespread PSA screening" may be self-evident, as the guidelines from the Ministry of Health, Labor and Welfare clearly state that "PSA testing is a useful test for the early diagnosis of prostate cancer" at the beginning. Already in 2005, the multicenter study in Sicily showed that PSA test is useful for early detection of prostate cancer, and it is possible to modulate most suitable timing for early diagnosis in individual patients with considering age and serum PSA. ${ }^{12}$

In the past era without PSA testing, a substantial proportion of prostate cancer patients were metastatic. In this sense, the current recognition that most prostate cancers have good prognoses could be only in the PSA era. However, if the mass media negatively reports on PSA screening for local residents, the significance of such results, including "widespread PSA screening" may be interpreted in a negative manner in many instances, which may lead to a significantly negative impact.
Based on the results in this study, $42.6 \%$ of newly diagnosed prostate cancer patients in Niigata Prefecture were discovered by implementing organized screening, suggesting that the medical practice and treatment of prostate cancer cannot be achieved without organized screening.

Looking at the metastatic cancer rate, $14.9 \%$ of the target patients in this study had metastatic cancer; however, this rate of metastatic cancer is still high compared to the United States. Metastatic cancer accounted for as high as $23 \%$ of newly diagnosed prostate cancer patients in the United States around 1980, prior to widespread use of PSA screening, which decreased to 5\% in 2007-2013 because of the widespread use of screening. Nevertheless, in response to the results of the PLCO study, the United States has been moving toward discontinuation of PSA screening and consequently the proportion of metastatic cancer increased to $8 \%$ in $2017 .^{13}$

According to the cancer registry data, the metastatic cancer rate of Japanese prostate cancer in 2019 was $17 \%,{ }^{14}$ and is similar to the present study. Since the metastatic cancer rate has been reported to decrease as PSA screening exposure increases, ${ }^{15}$ the reason why Japan has a higher ratio of metastatic cancers than in western countries is probably because exposure to PSA screening is still low.

Thus, there seems to be room in Japan to further promote screening and increase the proportion of early cancers detected. A high proportion of early cancers possibly increases the likelihood of cure for cancer, which is certain to improve treatment outcomes including survival.

However, for prostate cancer, it is true that there are a certain number that do not require treatment such as those with latent cancer. ${ }^{16}$ Therefore, PSA screening also has a risk of resulting in unnecessarily poor quality of life because overdiagnosis and overtreatment cannot be completely ruled out.

As a countermeasure for this, there is now the active surveillance approach, which is gradually gaining popularity. Moreover, a large-scale research study on active surveillance is ongoing, which has been sufficiently and safely conducted. ${ }^{17-19}$

Second, advances in diagnostic modalities are making it possible to properly select prostate biopsy targets. In the present study, $83.3 \%$ of the patients underwent MRI. Although our data could not evaluate whether MRI could have avoided unnecessary biopsy, it has been shown that 
MRI and targeted fusion prostate biopsy contribute to the diagnosis of clinically significant prostate cancer. ${ }^{20}$

These are the big difference from the early 2000s when overdiagnosis and overtreatment became a problem in the United States, and there is no concern that more widespread screening will lead to the same mistakes that were made previously in the United States.

Recently in the United States, there is movement toward reconsidering the withdrawal from PSA screening. The United States Preventive Services Task Force, which had recommended in 2012 to stop PSA screening in asymptomatic individuals of all ages, acknowledged a cancer mortality reduction effect in patients 55-69 years of age in 2018 and redefined the recommendation level to $\mathrm{C}$ (to be determined for each individual after obtaining informed consent after explaining the benefits and disadvantages). ${ }^{21}$

This is not the time to withdraw from PSA screening in Japan. The results of this survey in Niigata Prefecture are considered to clearly demonstrate that PSA screening plays a significant role in the medical practice and treatment of prostate cancer in Japan, but the spread of PSA screening is not yet sufficient.

Following international clinical practice, PSA screening should be further disseminated in Japan, and this survey seems to be useful information as basic data for this purpose.

\section{Limitations}

We acknowledge several weak points of our study. First, since the clinical stage and Gleason Score were assessed by several physicians, these data may be subjective and heterogeneous. Second, the triggers for the PSA measurement may be inaccurate due to patient declarations. Third, for the treatment outcomes have not been assessed, we cannot lead the conclusion that PSA screening is useful for reducing mortality.

\section{Conclusion}

The one-year all-case survey of newly diagnosed prostate cancer patients in Niigata Prefecture demonstrated that prostate cancer screening has greatly contributed to the current medical practice and treatment of prostate cancer in Japan. The proportion of metastatic cancer in newly diagnosed prostate cancer patients in Japan is still higher than that in the United States, suggesting that there is a requirement to disseminate PSA screening.

\section{Acknowledgments}

The authors deeply appreciate those who have diligently obtained clinical data: Toru Uehara, Tachikawa General Hospital; Yasuo Kitamura, Kido Hospital; Atsushi Yoshimizu, Saiseikai Niigata Hospital; Sadanobu Satomi, Joetsu General Hospital; Yoshiaki Kawakami, Niigata City General Hospital; Toshiki Tanikawa, Niigata Cancer Center Hospital; Motohiko Kimura, Niigata Medical Center; Shunji Wakatsuki, Niigata Prefectural Yoshida Hospital; Toshiyuki Itoi, Niigata Rinko Hospital; Bilim Vladimir, Kameda Daiichi Hospital; Tomotaka Toba, Sado General Hospital.

\section{Disclosure}

Dr Yoshihiko Tomita reports grants and/or personal fees from Ono Pharmaceuticals, BMS, Pfizer, Takeda, and Astellas, outside the submitted work. The authors report no other conflicts of interest in this work.

\section{References}

1. Hamashima C, Nakayama T, Sagawa M, Saito H, Sobue T. Japanese guideline for prostate cancer screening. Jpn J Clin Oncol. 2009;39:339-351. doi:10.1093/jjco/hyp025

2. Schröder FH, Hugosson J, Roobol MJ, et al. Screening and prostate cancer mortality: results of the European Randomized Study of Screening for Prostate Cancer (ERSPC) at 13 years of follow-up. Lancet. 2014;384:2027-2035. doi:10.1016/S0140-6736(14)60525-0

3. Hugosson J, Roobol MJ, Månsson M, et al. ERSPC investigators: a 16-yr follow-up of the European randomized study of screening for prostate cancer. Eur Urol. 2019;76:43-51. doi:10.1016/j.eururo.2019.02.009

4. Hugosson J, Carlsson S, Aus G, et al. Mortality results from the Göteborg randomized population-based prostate-cancer screening trial. Lancet Oncol. 2010;11:725-732. doi:10.1016/S1470-2045(10) 70146-7

5. Oberaigner W, Siebert U, Horninger W, et al. Prostate-specific antigen testing in Tyrol, Austria: prostate cancer mortality reduction was supported by an update with mortality data up to 2008. Int J Public Health. 2012;57:57-62. doi:10.1007/s00038-011-0266-4

6. The Japanese Urological Association. Screening guideline for prostate cancer Medical View. Tokyo, Japan. 2018. Japanese.

7. Andriole GL, Crawford ED, Grubb RL, et al. Mortality results from a randomized prostate-cancer screening trial. $N$ Engl J Med. 2009;360:1310-1319. doi:10.1056/NEJMoa0810696

8. Pinsky PF, Prorok PC, Yu K, et al. Extended mortality results for prostate cancer screening in the PLCO trial with median follow-up of 15 years. Cancer. 2017;123:592-599. doi:10.1002/cncr.30474

9. Ito K, Oki R, Sekine Y, et al. Screening for prostate cancer: history, evidence, controversies and future perspectives toward individualized screening. Int J Urol. 2019;26:956-970. doi:10.1111/iju.14039

10. Komatsubara S, Nishiyama T, Katagiri A, et al. [Screening of prostate cancer in Niigata prefecture]. $J$ Niigata Med Assoc. 2015;786:6-10. Japanese.

11. Niigata Prefectural Government. Results of prostate cancer screening; 2021. Available from: https:/www.kenko-niigata.com/material/files/ group/4/01_g_zenritsusengan.xlsx. Accessed November 15, 2021. Japanese.

12. Aragona F, Pepe P, Motta M, et al. Incidence of prostate cancer in Sicily: results of a multicenter case-findings protocol. Eur Urol. 2005;47:569-574. doi:10.1016/j.eururo.2004.11.007 
13. Siegel DA, O'Neil ME, Richards TB, Dowling NF, Weir HK. Prostate cancer incidence and survival, by stage and race/ethnicity United States, 2001-2017. MMWR Morb Mortal Wkly Rep. 2020;69:1473-1480. doi:10.15585/mmwr.mm6941a1

14. National Cancer Center Japan, Center for Cancer Control and Information Services. Cancer registry at cancer medical care cooperation base hospitals 2019 national report; 2021. Available from: https://ganjoho.jp/public/qa_links/report/hosp_c/pdf/2019_report.pdf. Accessed November 15, 2021.Japanese.

15. Kitagawa Y, Namiki M. Prostate-specific antigen-based population screening for prostate cancer: current status in Japan and future perspective in Asia. Asian J Androl. 2015;17:475-480. doi:10.4103/ 1008-682X.143756

16. Bell KJ, Del Mar C, Wright G, Dickinson J, Glasziou P. Prevalence of incidental prostate cancer: a systematic review of autopsy studies. Int J Cancer. 2015;137:1749-1757. doi:10.1002/ijc.29538
17. Bul M, Zhu X, Valdagni RT, et al. Active surveillance for low-risk prostate cancer worldwide: the PRIAS study. Eur Urol. 2013;63:597-603. doi:10.1016/j.eururo.2012.11.005

18. Mitsuzuka K, Koga H, Sugimoto M, et al. Current use of active surveillance for localized prostate cancer: a nationwide survey in Japan. Int J Urol. 2015;22:754-759. doi:10.1111/iju.12813

19. Kato T, Sugimoto M. Quality of life in active surveillance for early prostate cancer. Int J Urol. 2020;27:296-306. doi:10.1111/iju.14202

20. Pepe P, Garufi A, Priolo GD, et al. Is it time to perform only magnetic resonance imaging targeted cores? Our experience with 1032 men who underwent prostate biopsy. $J$ Urol. 2018;200:774-778. doi:10.1016/j.juro.2018.04.061

21. Grossman DC, Curry SJ, Owens DK, et al. Screening for prostate cancer: US preventive services task force recommendation statement. JAMA. 2018;319:1901-1913. doi:10.1001/jama.2018.3710

\section{Publish your work in this journal}

Research and Reports in Urology is an international, peer-reviewed, open access journal publishing original research, reports, editorials, reviews and commentaries on all aspects of adult and pediatric urology in the clinic and laboratory including the following topics: Pathology, pathophysiology of urological disease; Investigation and treatment of urological disease; Pharmacology of drugs used for the treatment of urological disease. The manuscript management system is completely online and includes a very quick and fair peer-review system, which is all easy to use. Visit http://www.dovepress.com/ testimonials.php to read real quotes from published authors. 